\title{
Cystic hemispheric medulloepithelioma
}

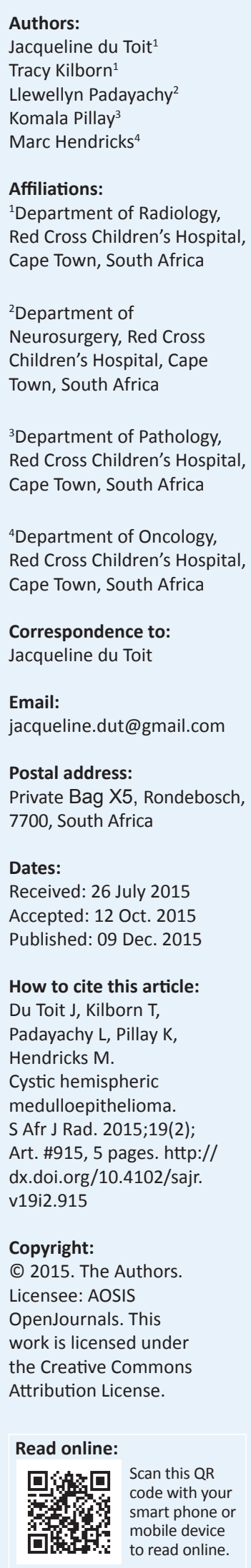

Medulloepithelioma is a rare primitive embryonal tumour of the central nervous system which typically occurs in early childhood. The histopathological appearance has been confused with primitive neuroectodermal tumours (PNETs), but medulloepitheliomas have a more aggressive clinical course. Imaging typically demonstrates a well-circumscribed, poorly or non-enhancing solid mass, with cyst formation being infrequent. We report the imaging findings in a case of a cystic hemispheric medulloepithelioma. The importance of considering this diagnosis is discussed in view of the propensity to aggressive recurrence in subtotal resection.

\section{Introduction}

Medulloepithelioma is a rare central nervous system (CNS) tumour of childhood with a very poor prognosis. Although the tumour has a variable appearance on neuroimaging, cyst formation is said to be infrequent. We report the imaging and histological findings of a predominantly cystic medulloepithelioma with an innocuous radiological appearance belying its aggressive behaviour.

\section{Case Report}

A five-year-old girl presented to the casualty department of our institution with a two-week history of progressive headache and vomiting. On examination she was generally well, with no demonstrable neurological deficit.

Computerised tomography (CT) of the brain (Figure 1) revealed a predominantly cystic mass in the right posterior parietal lobe, with the impression of a small nodular enhancing solid component medially. There was mass effect with midline shift to the left and contralateral ventricular dilatation.

Magnetic resonance imaging (MRI) of the brain (Figure 2) confirmed the predominantly cystic nature of the well-circumscribed mass within the right parietal lobe, with minimal surrounding peritumoral oedema. The cystic component was T1 hypo- and T2 hyperintense. Images post gadolinium showed both peripheral rim enhancement and a small nodular enhancing solid component medially, abutting the lateral ventricle. Neither the cystic nor the solid components of the mass showed diffusion restriction. Neovascularity, haemorrhage, calcification, drop metastases and leptomeningeal spread were absent.

A right parieto-occipital craniotomy with subtotal resection was performed. Histological sections (Figure 3) showed a primitive neuro-ectodermal tumour with a heterogeneous appearance and cystic change.

Areas of primitive embryonal cells alternated with areas showing trabecular, papillary and tubular architecture, with variable amounts of eosinophilic matrix material resembling basement membrane-like material. Mitotic figures were brisk in the more primitive areas. A small focus of necrosis was also seen.

The pan-keratin marker MNF-116 was strongly positive in the tubulopapillary areas and there was focal positivity with AE1/AE3 (pan-keratin marker and epithelial membrane antigen). Collagen IV highlighted the basement membrane-type material bordering the trabecular areas. These areas also showed focal weak glial fibrillary acidic protein (GFAP) positivity. Neuronal markers (neurofilament and synaptophysin) were negative.

According to the World Health Organization (WHO) 2007 classification, the overall features were compatible with an embryonal tumour displaying evidence of ependymal and glial differentiation consistent with a diagnosis of medulloepithelioma (Figure 3). 

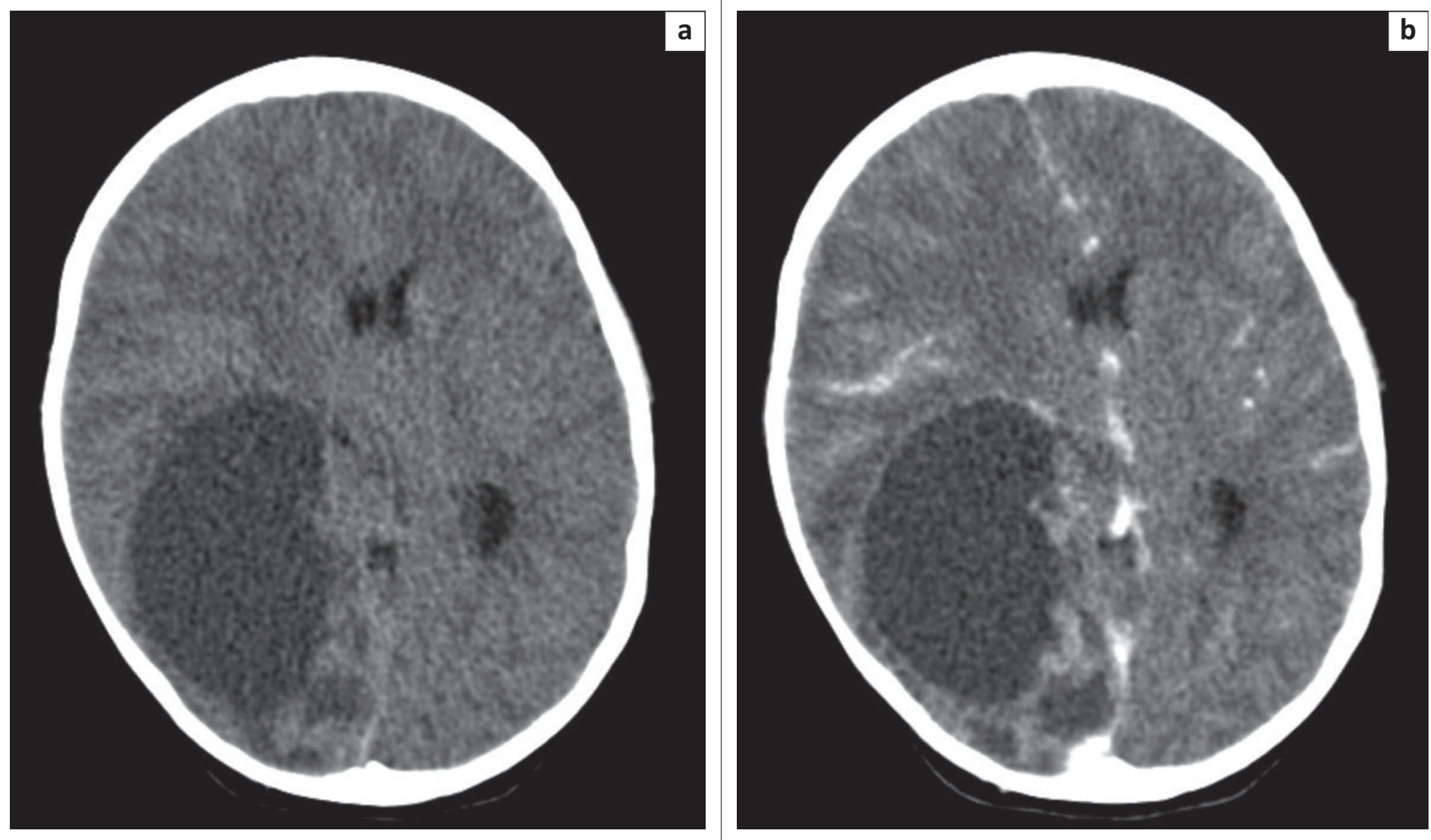

FIGURE 1: CT brain at presentation. Pre (a) and post (b) contrast axial CT images demonstrate a cyst in the right parietal lobe with a nodular enhancing component at its medial margin. There is associated right hemispheric swelling with midline shift to the left.

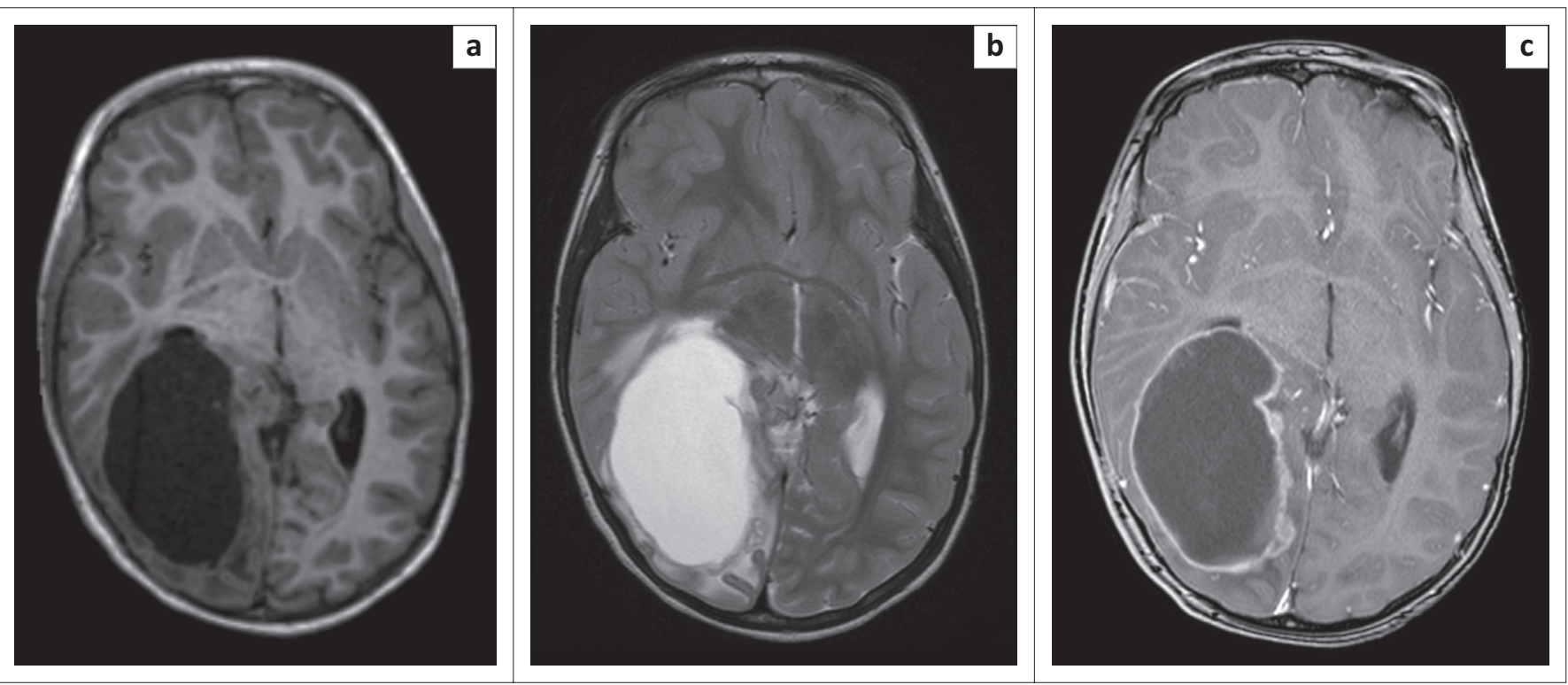

FIGURE 2: MRI brain at presentation. T1 (a) and T2 (b) axial MRI reveals a predominantly cystic mass in the right posterior parietal lobe with associated mass effect and contralateral ventricular dilatation. There is only minimal peri-tumoral oedema. The T1 post contrast image (c) shows peripheral rim-enhancement and a small nodular solid component medially. There is no significant neovascularity and no evidence of haemorrhage.

The patient was subsequently commenced on chemotherapy comprising high-risk medulloblastoma/supratentorial primitive neuroectodermal tumour (PNET) protocol, which is JOE-e (Vincristine IV, Carboplatin IV, Etoposide IV and Etoposide PO) alternating with OPEC (Vincristine IV, Cisplatin IV, Etoposide IV and Cyclophosphamide IV).
Follow-up imaging six weeks post-surgery, on chemotherapy, showed an increase in the size of the solid enhancing tumour component (Figure 4). A repeat craniotomy with gross total resection was performed, followed by radiotherapy and an intensive six-week cycle of chemotherapy. Repeat imaging is awaited. 

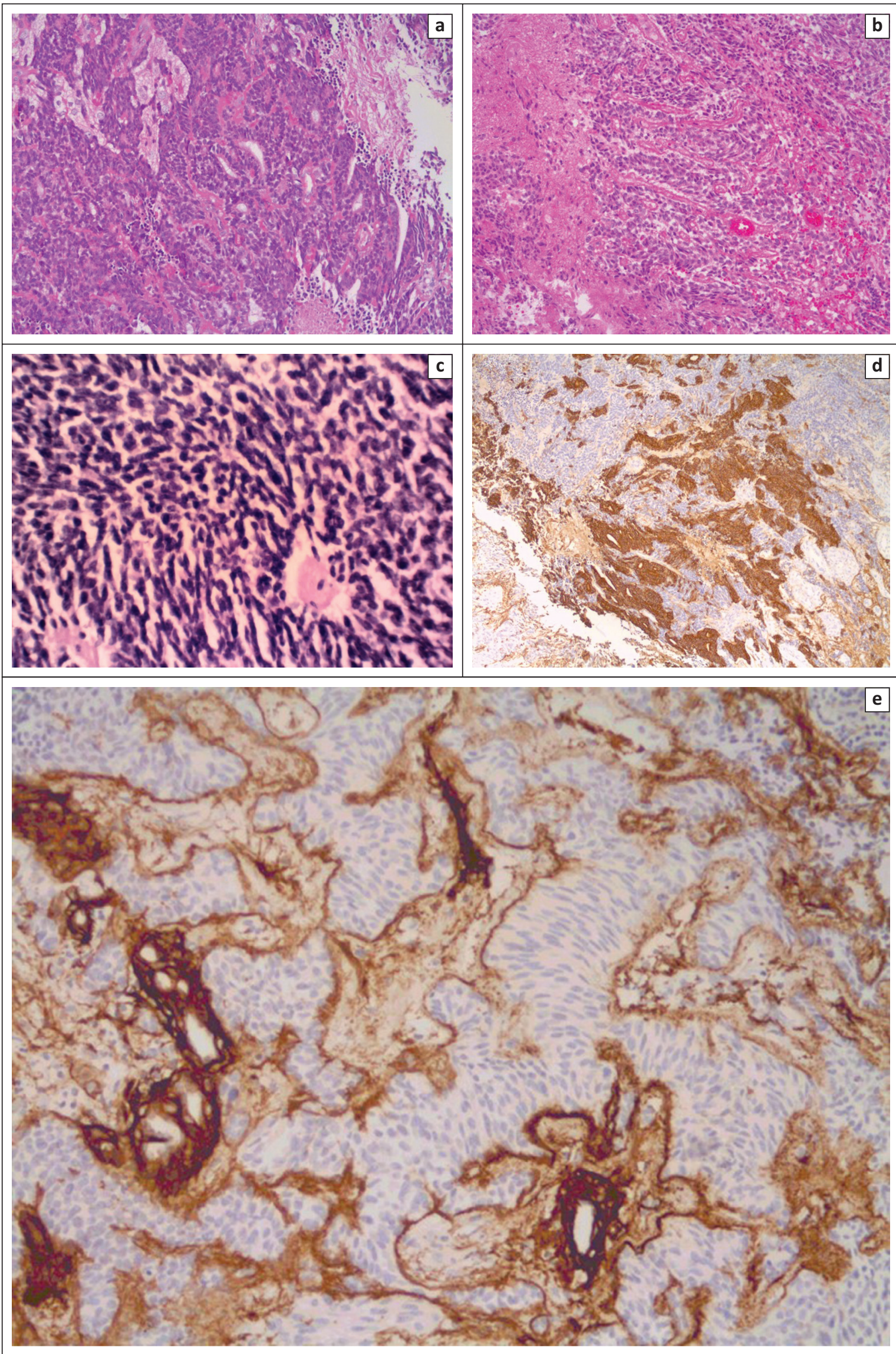

FIGURE 3: Tumour biopsy histology Haematoxylin and eosin sections showing an embryonal tumour with neuronal and ependymal differentiation (a), trabecular areas displaying basement membrane deposition (b), and a primitive embryonal component with Homer-Wright rosettes (c). Immunohistochemistry shows positivity with the epithelial marker AE1/AE3 (d) and confirms collagen IV positive basement membrane material (e). 


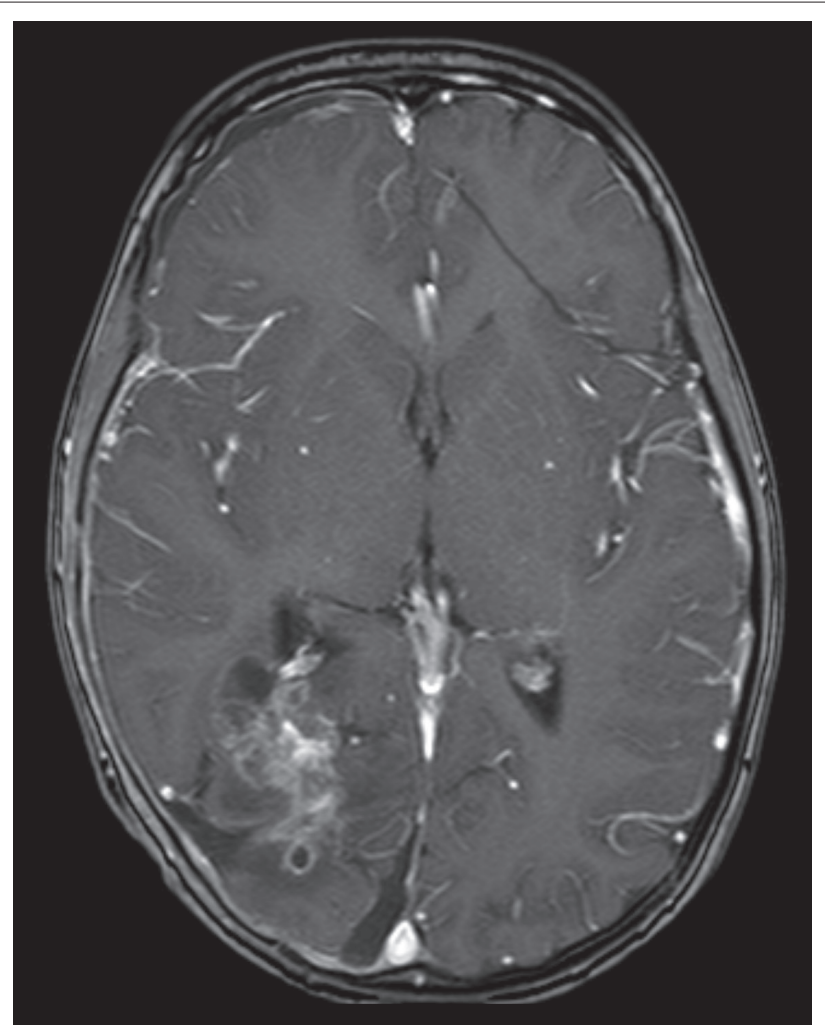

FIGURE 4: Follow-up MRI brain six weeks post-surgery, on chemotherapy. An axial T1 post-contrast image shows interval increase in the size of the solid tumour component.

\section{Discussion}

First described by Bailey and Cushing in 1926, CNS medulloepitheliomas are rare, highly malignant embryonal tumours which tend to occur in early childhood, usually between the ages of 6 months and 5 years. ${ }^{1,2,3}$ The prognosis is dismal, with a mean survival of 8.5 months from diagnosis. ${ }^{2,4}$

Interestingly, a histologically similar tumour, the intraorbital medulloepithelioma, which usually arises within the ciliary body, exhibits a much less malignant course, with excellent long-term survival. The difference in biological behaviour is unexplained. $^{2}$

A review of the literature reveals relatively few descriptions of the neuroimaging characteristics of these tumours, with MRI findings only having been reported in a handful of cases.,

Despite a variable radiological appearance, most cases are isodense or hypodense on CT and seldom display contrast enhancement as described in our case. ${ }^{2}$ The tumours tend to be large at the time of presentation, with extensive haemorrhagic and necrotic components. ${ }^{4,6}$ Hyperdense and partially cystic lesions have also been described.,56 Our patient demonstrated a predominantly cystic mass with a small peripheral nodule, a similar appearance to that in the first reported case on $\mathrm{CT}^{3}$
In the few reported cases, MRI has shown a solid mass predominantly hypointense on T1-WI, hyperintense on T2WI and rarely displaying contrast enhancement. This lack of enhancement is said to distinguish medulloepitheliomas from PNETs, which enhance avidly and are one of the main differential diagnoses in this clinical setting. ${ }^{2}$ Our case of a predominantly cystic lesion with a peripheral and enhancing nodular component has not been reported previously. Gadolinium enhancement is more commonly demonstrated in the case of tumour recurrence, likely indicating the more aggressive nature of recurrent disease as was also seen in the follow-up imaging of our patient. ${ }^{2}$ At the time of tumour progression, CNS medulloepithelioma is also prone to subarachnoid dissemination.

The diverse imaging appearance can be explained by the variable differentiation seen histologically. ${ }^{1,2}$ These tumours contain structures that resemble the primitive neural tube and approximately half of the cases exhibit the capacity for divergent differentiation into astroglial, ependymal or neuronal cell lines. Medulloepitheliomas are classified as WHO Grade IV embryonal tumours, but unlike other PNETS they have a dismal prognosis.

The tumour responds poorly to treatment - gross total resection and radiotherapy may provide some benefit. ${ }^{2}$ Post-operative scanning is needed to alert the neurosurgeon to the need for early intervention as all cases of subtotal resection reported within the literature went on to early progression, as in our case.

\section{Conclusion}

CNS medulloepithelioma is a rare tumour, but it should be considered in the differential diagnosis of a cystic hemispheric mass since the innocuous appearance belies its locally aggressive behaviour and a high incidence of recurrence post subtotal resection.

\section{Acknowledgements Competing interests}

The authors declare that they have no financial or personal relationships which may have inappropriately influenced them in writing this article.

\section{Authors' contributions}

J.d.T. (Red Cross Children's Hospital) and T.K. (Red Cross Children's Hospital) analysed the imaging, conducted a literature review and wrote the original manuscript. L.P. (Red Cross Children's Hospital) provided the clinical and operative details. K.P. (Red Cross Children's Hospital) performed the histopathological analysis and provided the relevant histology slides. M.H. (Red Cross Children's Hospital) provided clinical and management information. All authors were involved in final editing and checking. 


\section{References}

1. Bailey $\mathrm{P}$, Cushing $\mathrm{H}$. A classification of tumours of the Glioma group on histogenetic basis with a correlated study of progress. Philadelphia: JB Lippincott, 1926; p. 54-56.

2. Molloy PT, Yachnis AT, Rorke LB, et al. Central nervous system medulloepithelioma: A series of eight cases including two arising in the pons. J Neurosurg. 1996;84:430436. PMID: 8609554, http://dx.doi.org/10.3171/jns.1996.84.3.0430
3. Grainger RD, Allison DJ, editors. Diagnostic radiology. 5th edn. Churchill Livingston Elsevier, 2008; p. 1684.

4. Poot RD. Medulloepithelioma: First CT images. Neuroradiology. 1986;28:286 PMID: 3725021, http://dx.doi.org/10.1007/BF00548211

5. Pang LM, Roebuck DJ, Ng HK, Chan YL. Sellar and suprasellar medulloepithelioma. Pediatr Radiol. 2001;31:594-596. PMID: 11550774, http://dx.doi.org/10.1007/ s002470100495

6. Sundaram C, Vydehi B, Reddy J, Reddy AK. Medulloepithelioma: A case report. Neurol India. 2003;51:546-547. PMID: 14742946. 